\title{
Gametocidal chromosomes enhancing chromosome aberration in common wheat induced by 5-azacytidine
}

\author{
W.-Y. Su*, W.-W. Cong*, Y.-J. Shu, D. Wang, G.-H. Xu, C.-H. Guo \\ Key Laboratory of Molecular Cytogenetics and Genetic Breeding of \\ Heilongjiang Province, College of Life Science and Technology, \\ Harbin Normal University, Harbin, Heilongjiang Province, China \\ *These authors contributed equally to this study. \\ Corresponding author: C.-H. Guo \\ E-mail: kaku_2008@163.com
}

Genet. Mol. Res. 12 (3): 2227-2233 (2013)

Received December 21, 2012

Accepted March 12, 2013

Published July 8, 2013

DOI http://dx.doi.org/10.4238/2013.July.8.4

\begin{abstract}
The gametocidal (Gc) chromosome from Aegilops spp induces chromosome mutation, which is introduced into common wheat as a tool of chromosome manipulation for genetic improvement. The Gc chromosome functions similar to a restriction-modification system in bacteria, in which DNA methylation is an important regulator. We treated root tips of wheat carrying Gc chromosomes with the hypomethylation agent 5-azacytidine; chromosome breakage and micronuclei were observed in these root tips. The frequency of aberrations differed in wheat containing different Gc chromosomes, suggesting different functions inducing chromosome breakage. Gc chromosome 3C caused the greatest degree of chromosome aberration, while Gc chromosome $3 \mathrm{C}^{\mathrm{SAT}}$ and $2 \mathrm{C}$ caused only slight chromosome aberration. Gc chromosome $3 \mathrm{C}$ induced different degrees of chromosome aberration in wheat varieties Triticum aestivum
\end{abstract}


var. Chinese Spring and Norin 26, demonstrating an inhibition function in common wheat.

Key words: Gametocidal chromosomes; Aegilops cylindrica; Aegilops triuncialis; 5-Azacytidine; Chromosome aberration

\section{INTRODUCTION}

Wheat (Triticum spp), the second most produced cereal after maize, is one of the most important food crops in the world, and its production has a significant influence on economic development. However, the domestication and modern plant breeding of wheat have presumably narrowed its genetic base, which may jeopardize future crop improvement. Many species are closely related to wheat, and they display a diversity greater than that in wheat, which may provide plant breeders with a broad pool of potentially useful genetic resources.

Many alien chromosomes from related wild species, such as those in the genus Aegilops, have been introduced into common wheat. Some alien chromosomes have caused chromosome breakage of the host genome in the gametes that lack them, and such chromosomes are called gametocidal (Gc) chromosomes, also known as cuckoo chromosomes (Endo, 1982). Previous research has shown that Gc chromosome can cause sterility in both male and female gametes that lack the chromosomes. When the Gc action is intense, gametophytes without the alien chromosome may experience severe chromosome breakage, and the gametes become sterile (Miller et al., 1982). This response results in exclusive transmission of the alien chromosome, such as in the common wheat cultivar 'Chinese Spring' (CS) with Gc chromosomes 3C from Aegilops triuncialis, 4S from A. longissima, and A. sharonensis (Finch et al., 1984; Endo, 1988; Shi and Endo, 1999). When the Gc action is mild, gametophytes without the alien chromosome display only slight chromosome damage. Then, the gametes are fertilized and develop into plants with chromosome aberrations, for example, CS carrying Gc chromosome $2 \mathrm{C}$ from $A$. cylindrica (Endo, 1988).

In Gc chromosome action, chromosome aberrations induced by Gc chromosomes cause some chromosome mutations, such as deletions, translocations, and rearrangements. These chromosome variations could be transmitted to progeny, and chromosome variation would be stable in the offspring. Therefore, Gc chromosomes are widely used for genetic improvement in wheat through chromosome engineering - for example, production of deletion stocks in common wheat (Endo, 1990; Endo and Gill, 1996) or rearrangement of alien chromosomes added to common wheat. Thus far, many relatives of wheat have been described, and their genomes have been introduced into the wheat genome via Gc action to improve wheat, such as barley, Secale cereale, Thinopyrum elongatum, Leymus racemosus, Dasypyrum villosum, Leymus mollis, Agropyron cristatum (Endo et al., 1994; Shi and Endo, 1999, 2000; Masoudi-Nejad et al., 2002). These alien genomes have been introgressed into the host genome via chromosome rearrangement induced by Gc chromosomes, in which the alien chromosome transfers its effect and greatly broadens genetic resources for wheat improvement (Endo, 2007).

Although Gc chromosomes have been broadly used in chromosome manipulation 
in wheat, the mechanism of its inducing chromosome mutation remains unknown. de las Heras et al. (2001) treated wheat with the DNA demethylation reagent 5-azacytidine (5$\mathrm{AC}$ ) that contained Gc chromosome 4SL. Chromosome aberration was identified in the cells of the root tip, suggesting that a decline in DNA methylation would enhance Gc effects. However, the detailed regulation model of Gc action by DNA methylation was unclear. Investigation of Gc chromosome action has revealed that it functions similarly to the segregation distorter system in fruit fly and the t-haplotype in mice. Tsujimoto (2005) published a new model, which is similar to "restriction-modification systems" in bacteria, to explain the Gc action mechanism. Gc chromosomes transferred into a host genome have both chromosome break-inducing and -suppressing functions. The chromosome break-inducing function of Gc chromosomes is active in pollen mitoses. Chromosome break-suppressing function is induced in gametes with Gc chromosomes, which protect the chromosome from damage. However, gametes lacking the Gc chromosome cannot achieve protected function and display damaged chromosomes with abnormalities. This phenomenon has been observed in many addition lines of common wheat with Gc chromosomes, but the mechanism of Gc function has remained unclear. During gamete development, epigenetic changes regulate all changes such as gametes process, egg fertilization, and egg cell development (Burn et al., 1993). Therefore, the Gc process is regulated by DNA methylation.

Therefore, in this study, we used the demethylation reagent 5-AC to treat an addition line of common wheat with mild Gc chromosomes. It simulated the DNA demethylation phenomenon of the gamete formation, and the mechanism by which the Gc chromosome induces chromosome aberration was determined.

\section{MATERIAL AND METHODS}

\section{Plant materials}

Common wheat cultivars CS and 'Norin 26' (N26) were obtained from the National BioResource Project. Gc chromosomes $2 \mathrm{C}$ and $3 \mathrm{C}$ were isolated from A. cylindrical and $A$. triuncialis, respectively, and the Gc chromosome $3 \mathrm{C}^{\mathrm{SAT}}$ was also identified with an unknown satellite from $A$. triuncialis. These Gc chromosome disomic addition lines were developed by Professor Endo (National BioResource Project - Wheat, Japan) and included the following: Chinese spring-Gc chromosome $2 \mathrm{C}$ disomic addition line (CS-2C2C), Chinese springGc chromosome 3C disomic addition line (CS-3C3C), Chinese spring-Gc chromosome 3C $\mathrm{C}^{\mathrm{SAT}}$ disomic addition line $\left(\mathrm{CS}-3 \mathrm{C}^{\mathrm{SAT}} 3 \mathrm{C}^{\mathrm{SAT}}\right.$ ), and $\mathrm{N} 26-\mathrm{Gc}$ chromosome $3 \mathrm{C}$ disomic addition line (N26-3C3C).

\section{Cytogenetic analysis}

All plants were placed on water-soaked filter paper on Petri dishes at $4^{\circ} \mathrm{C}$ for 1 day. After pre-chilling, the filter papers were replaced with fresh ones. Seeds were germinated on Petri dishes with filter paper moistened in $100 \mu \mathrm{M} \mathrm{5-AC}$ and distilled water (control). These seeds were then incubated in the dark at $23^{\circ} \mathrm{C}$ for $2-3$ days to induce germination. The filters were replaced daily. After germination, root tips from each dish were collected 
and fixed in the Carnoy fluid for $24 \mathrm{~h}$ and stored in $70 \%$ alcohol at $4{ }^{\circ} \mathrm{C}$ for slide preparation. Root tips were treated with $1 \mathrm{M} \mathrm{HCl}$ and stained with fuchsine acetum (Sigma, St. Louis, MO, USA).

All slides were examined microscopically, first at low-power magnification and then at high power (40 or 100X). Chromosomal aberrations were scored, and the micronucleus test (MNT) was performed. The treatment procedure, slide preparation, and scoring were carried out according to standard protocols described by Michael (1993) and Yi et al. (2005). Only cells with intact cytoplasm in which the micronucleus size was maximally one-third that of the main nucleus, had a similar color, rounded or oval shape, and texture with the same refraction, and lay in the same focal plane as that of the nucleus were recorded. All suspected micronuclei were assessed by a third scorer and discussed until a consensus was reached. The frequency of chromosomal aberration and micronucleus formation were expressed as the number of cells with chromosomal aberration in 3 replications. All data were collected and analyzed with $\mathrm{R}$ platform (http://www.r-project.org/).

\section{RESULTS}

\section{Chromosome aberration induced by Gc chromosomes and 5-AC treatment}

Water-germinated CS and N26 root tips showed a low level of chromosome aberration (see Table 1). When the root tips of CS and N26 were treated with 5-AC, 4-5-fold increase in chromosome aberration occurred, such as chromosome bridges (Figure 1A). The frequency of aberrations in root tips with CS- $2 \mathrm{C} 2 \mathrm{C}, \mathrm{CS}-3 \mathrm{C} 3 \mathrm{C}, \mathrm{CS}-3 \mathrm{C}^{\mathrm{SAT}} 3 \mathrm{C}^{\mathrm{SAT}}$, and $\mathrm{N} 26-3 \mathrm{C} 3 \mathrm{C}$ germinated in water was similar to that observed in CS and N26, and no fragments occurred. In contrast, treatment of the root tips containing $\mathrm{Gc}$ chromosomes $2 \mathrm{C}, 3 \mathrm{C}$, and $3 \mathrm{C}^{\mathrm{SAT}}$ with 5-AC resulted in chromosome fragmentation (Figure 1B, C, and D) and chromosome bridges. Chromosome fragments were the predominant chromosome aberration. The frequency of chromosome mutation increased from less than $1 \%$ to $6.67 \%, 14.01 \%, 7.65 \%$, and $8.74 \%$ in $\mathrm{CS}-2 \mathrm{C} 2 \mathrm{C}$, CS$3 \mathrm{C} 3 \mathrm{C}, \mathrm{CS}-3 \mathrm{C}^{\mathrm{SAT}} 3 \mathrm{C}^{\mathrm{SAT}}$, and $\mathrm{N} 26-3 \mathrm{C} 3 \mathrm{C}$ respectively (see Table 1), which were more than in CS and $\mathrm{N} 26$ treatment with 5-AC.

\begin{tabular}{|c|c|c|c|}
\hline Materials & Total cells observed & Aberrations & Aberration rate $(\%)$ \\
\hline CS (control) & 201 & 1 & 0.50 \\
\hline $\mathrm{CS}(5-\mathrm{AC})$ & 290 & 7 & 2.41 \\
\hline N26 (control) & 214 & 1 & 0.47 \\
\hline $\mathrm{N} 26(5-\mathrm{AC})$ & 282 & 6 & 2.13 \\
\hline $\mathrm{CS}-2 \mathrm{C} 2 \mathrm{C}$ (control) & 242 & 2 & 0.83 \\
\hline $\mathrm{CS}-2 \mathrm{C} 2 \mathrm{C}(5-\mathrm{AC})$ & 195 & 13 & $6.67 * *$ \\
\hline CS-3C3C (control) & 316 & 3 & 0.95 \\
\hline $\mathrm{CS}-3 \mathrm{C} 3 \mathrm{C}(5-\mathrm{AC})$ & 207 & 29 & $14.01 * *$ \\
\hline CS-3C $\mathrm{C}^{\mathrm{SAT}} 3 \mathrm{C}^{\mathrm{SAT}}$ (control) & 237 & 2 & 0.84 \\
\hline $\mathrm{CS}-3 \mathrm{C}^{\mathrm{SAT}} 3 \mathrm{C}^{\mathrm{SAT}}(5-\mathrm{AC})$ & 170 & 13 & $7.65 * *$ \\
\hline N26-3C3C (control) & 222 & 2 & 0.90 \\
\hline $\mathrm{N} 26-3 \mathrm{C} 3 \mathrm{C}(5-\mathrm{AC})$ & 206 & 18 & $8.74 * *$ \\
\hline
\end{tabular}

$* *$ Signature level of 0.01 . 


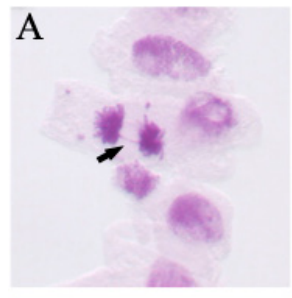

$\mathrm{D}$

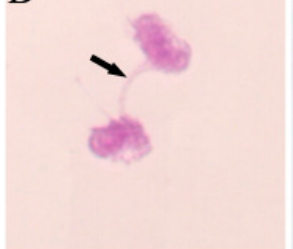

B

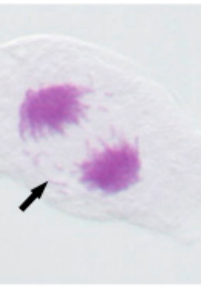

$\mathrm{E}$

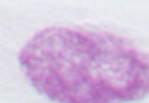

$\mathrm{C}$

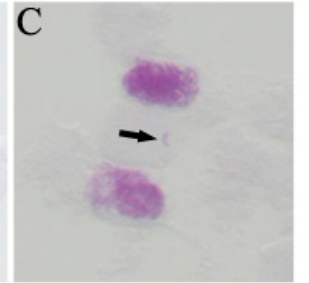

F

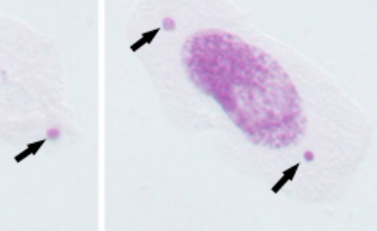

Figure 1. Chromosome aberration forms in root tips of common wheat carrying different Gc chromosomes. A. Bridge in anaphase; B. lots of fragments in anaphase; C. fragment in telophase; D. bridge in telophase; E. micronucleus in interphase; $\mathbf{F}$. double micronuclei in interphase.

\section{Appearance of micronuclei in Gc chromosomes inducing chromosome mutation}

An increased number of micronuclei (Figure 1E and F) were observed in wheat carrying Gc chromosomes treated with $100 \mu \mathrm{M}$ 5-AC compared with those in controls. The difference was significant, and different Gc chromosomes induced different levels of micronuclei. The results showed that $\mathrm{Gc}$ chromosome $3 \mathrm{C}$ had the strongest $\mathrm{Gc}$ action and produced the most micronuclei, whereas $\mathrm{Gc}$ chromosomes $3 \mathrm{C}^{\mathrm{SAT}}$ and $2 \mathrm{C}$ displayed mild $\mathrm{Gc}$ action (Figure 2). In addition, the genotype of the host wheat played an important role in chromosome mutation induction - for example, chromosome aberration induced by Gc chromosome $3 \mathrm{C}$ in $\mathrm{CS}$ was stronger than that induced in N26. The results suggested that an inhibitor of Gc action existed in N26 but was absent in CS.

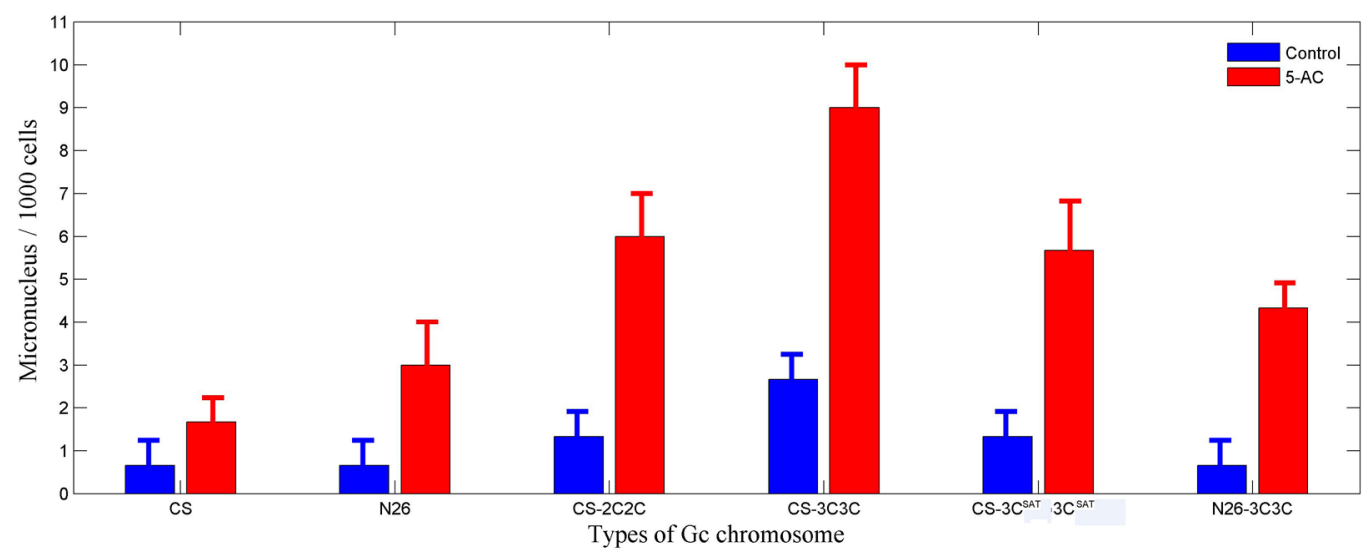

Figure 2. Mean micronucleus number/1000 cells in common wheat carrying different Gc chromosomes. 


\section{DISCUSSION}

5-AC is a demethylation reagent that causes a loss of methylated cytosine residues, which serve as cell cycle checkpoint blocks. It induces chromosome mutations in cells, but this function is mild, as shown in CS and N26, which displayed few chromosome mutations. The Gc chromosome from Aegilops induced chromosome breakage in gametes with a common wheat background (such as CS and N26), and the chromosome aberrations in gametes without Gc chromosomes included telocentric, acrocentric, deleted, dicentric, ringed, and translocated chromosomes, among others (Endo, 1988). However, chromosome aberration was absent in normal tissues and cells, such as root tip cells, demonstrating that Gc chromosomes did not function in normal cells. Using a Gc chromosome function model, Tsujimoto (2005) implemented a bacterial restriction-modification system to explain Gc chromosome function. DNA methylation was an important regulation factor in this system. Therefore, we used the hypomethylation agent 5-AC to model the change in DNA methylation during gametogenesis. Treatment of CS or N26 seeds carrying Gc chromosomes $3 \mathrm{C}, 3 \mathrm{C}^{\mathrm{SAT}}$, or $2 \mathrm{C}$ with 5-AC initiated breakage fusion bridge cycles in the root tips. These breakage fusion bridges resulted in chromosome fragmentation, chromosome bridges, and an aberration style similar to the tendency to fuse and form dicentric chromosomes seen in single chromatid segmentsbroken chromosome ends (de las Heras et al., 2001).

Among these $\mathrm{Gc}$ chromosomes, $3 \mathrm{C}$ had the most intense Gc action, inducing the most chromosome aberration and micronuclei with treatment of 5-AC in CS. However, 2C and $3 \mathrm{C}^{\mathrm{SAT}}$ induced mild chromosome mutation in CS, suggesting that the intensity of Gc chromosomes, which were isolated from different Aegilops species, is variable. For example, Gc chromosome $3 \mathrm{C}$ was isolated from $A$. triuncialis, which exerted severe $\mathrm{Gc}$ action. Gc chromosome $2 \mathrm{C}$, which was isolated from A. cylindrica, induced mild Gc action. Gc chromosomes $3 \mathrm{C}$ and $3 \mathrm{C}^{\mathrm{SAT}}$ were both derived from the same $A$. triuncialis plant, but they induced chromosome mutation differently. That of $3 \mathrm{C}$ was more intense than that of $3 \mathrm{C}^{\mathrm{SAT}}$. $\mathrm{Gc}$ chromosome $3 \mathrm{C}$ is a derivative of $3 \mathrm{C}^{\mathrm{SAT}}$ or $3 \mathrm{C}^{\mathrm{SAT}}$ is a rearranged $3 \mathrm{C}$ that acquired its satellite from one of the $A$. triuncialis satellite chromosomes. The chromosome mutation results suggested that the satellite chromosome would have a Gc inhibitor gene or Gc inhibitor-like function, which would reduce or inhibit Gc action. However, Gc chromosome $3 \mathrm{C}$ induced chromosome aberration in CS and N26 differently - that induced in CS was more intense than that induced in N26. The results indicated that $\mathrm{N} 26$, but not $\mathrm{CS}$, carried a genetic factor that could suppress the Gc function of Gc chromosome 3C (Endo, 2007).

The MNT has been widely applied to evaluate chromosomal instability in animals and plants exposed to chemical reagents with genetic toxicology, for example, 5-AC. Furthermore, the induced micronucleus frequency, as an expression of chromosome mutation sensitivity, is viewed as a possible means of investigating Gc chromosome intensity. High Gc intensity, such as that in $\mathrm{CS}-3 \mathrm{C} 3 \mathrm{C}$, is associated with a greater frequency of micronucleus. When Gc action was mild, as in $\mathrm{CS}-2 \mathrm{C} 2 \mathrm{C}$ and $\mathrm{CS}-3 \mathrm{C}^{\mathrm{SAT}} 3 \mathrm{C}^{\mathrm{SAT}}$, fewer micronuclei were seen. Additionally, the MNT was performed more easily than were other chromosome mutation detection methods such as the chromosome aberration test. Therefore, it was more suitable for chromosome mutation detection. In our research, MNT detection results were consistent with chromosome aberration detection, which suggested that the MNT can be used in intensity detection for various types of $\mathrm{Gc}$ chromosomes. 
In summary, Gc chromosomes induced chromosome aberration in the gametogenesis of common wheat. When root tips were treated with 5-AC, the Gc chromosomes functioned, and chromosome aberration occurred in host cells. Furthermore, Gc action was regulated by the combination of $\mathrm{Gc}$ chromosome and host genotype, and its functional mechanism will be revealed in the future.

\section{ACKNOWLEDGMENTS}

Research supported by the National Basic Research Program of China (\#2011CB111500), the International S\&T Cooperation Program of China (\#2009DFA32470), the Natural Science Foundation of Heilongiiang Province of China (\#QC2011C108), the Science and Technology Innovative Research Team in Higher Educational Institutions of Heilongjiang Province (\#2010TD10), and the Innovation Research Group of Harbin Normal University (\#KJTD201102). Materials containing Gc chromosomes were supplied by the National BioResource Project (NBRP)-Wheat.

\section{REFERENCES}

Burn JE, Bagnall DJ, Metzger JD, Dennis ES, et al. (1993). DNA methylation, vernalization, and the initiation of flowering. Proc. Natl. Acad. Sci. U. S. A. 90: 287-291.

de las Heras JI, King IP and Parker JS (2001). 5-Azacytidine induces chromosomal breakage in the root tips of wheat carrying the cuckoo chromosome 4S(L) from Aegilops sharonensis. Heredity 87: 474-479.

Endo TR (1982). Gametocidal chromosomes of three Aegilops species in common wheat. Can. J. Genet. Cytol. 24: 201206.

Endo TR (1988). Induction of chromosomal structural changes by a chromosome of Aegilops cylindrica L. in common wheat. J. Hered. 79: 366-370.

Endo TR (1990). Gametocidal chromosomes and their induction of chromosome mutations in wheat. Jpn. J. Genet. 65: $135-152$.

Endo TR (2007). The gametocidal chromosome as a tool for chromosome manipulation in wheat. Chromosome Res. 15: 67-75.

Endo TR and Gill BS (1996). The deletion stocks of common wheat. J. Hered. 87: 295-307.

Endo TR, Yamamoto M and Mukai Y (1994). Structural changes of rye [Secale cereale] chromosome 1R induced by a gametocidal chromosome. Jpn. J. Genet. 69: 13-19.

Finch RA, Miller TE and Bennett MD (1984). "Cuckoo" Aegilops addition chromosome in wheat ensures its transmission by causing chromosome breaks in meiospores lacking it. Chromosoma 90: 84-88.

Masoudi-Nejad A, Nasuda S, McIntosh RA and Endo TR (2002). Transfer of rye chromosome segments to wheat by a gametocidal system. Chromosome Res. 10: 349-357.

Michael F (1993). The cytokinesis-block micronucleus technique: A detailed description of the method and its application to genotoxicity studies in human populations. Mutat. Res. / Fund. Mol. Mech. Mutagenesis 285: 35-44.

Miller TE, Hutchinson J and Chapman V (1982). Investigation of a preferentially transmitted Aegilops sharonensis chromosome in wheat. Theor. Appl. Genet. 61: 27-33.

Shi F and Endo TR (1999). Genetic induction of structural changes in barley chromosomes added to common wheat by a gametocidal chromosome derived from Aegilops cylindrica. Genes Genet. Syst. 74: 49-54.

Shi F and Endo TR (2000). Genetic induction of chromosomal rearrangements in barley chromosome $7 \mathrm{H}$ added to common wheat. Chromosoma 109: 358-363.

Tsujimoto H (2005). Gametocidal Genes in Wheat as the Inducer of Chromosome Breakage. In: Frontiers of Wheat Bioscience (Tsunewaki K, ed.). Memorial Issue, Wheat Information Service No. 100. Kihara Memorial Yokohama Foundation, Yokohama, 33-48.

Yi H, Liu J and Zheng K (2005). Effect of sulfur dioxide hydrates on cell cycle, sister chromatid exchange, and micronuclei in barley. Ecotoxicol. Environ. Saf. 62: 421-426. 